\title{
Impact of the malaxation temperature on the phenolic profile of $c v$. Cobrançosa olive oils and assessment of the related health claim
}

\author{
Ítala M.G. Marx ${ }^{\mathrm{a}, \mathrm{b}}$, Susana Casal ${ }^{\mathrm{b}, *}$, Nuno Rodrigues ${ }^{\mathrm{a}}$, Teresa Pinho ${ }^{\mathrm{b}}$, Ana C.A. Veloso ${ }^{\mathrm{c}, \mathrm{d}}$, \\ José A. Pereira ${ }^{\mathrm{a}}$, António M. Peres ${ }^{\mathrm{a}, *}$ \\ ${ }^{a}$ Centro de Investigação de Montanha (CIMO), ESA, Instituto Politécnico de Bragança, Campus Santa Apolónia, 5300-253 Bragança, Portugal \\ ${ }^{\mathrm{b}}$ LAQV/REQUIMTE, Laboratory of Bromatology and Hydrology, Faculty of Pharmacy, University of Porto, Rua de Jorge Viterbo Ferreira, 228, 4050-313 Porto, Portugal \\ c Instituto Politécnico de Coimbra, ISEC, DEQB, Rua Pedro Nunes, Quinta da Nora, 3030-199 Coimbra, Portugal \\ ${ }^{\mathrm{d}}$ CEB - Centre of Biological Engineering, University of Minho, Campus de Gualtar, 4710-057 Braga, Portugal
}

\section{A R T I C L E I N F O}

\section{Keywords:}

Virgin olive oil

Extraction conditions

Health claim

Phenolic composition

Secoiridoids acid hydrolysis

\begin{abstract}
A B S T R A C T
Phenolic compounds contribute to the bioactive properties of olive oil. However, olive oils can only support a health claim concerning the protection against oxidative stress depending on the polyphenolic concentration, requiring effective measures during extraction to preserve/enhance their concentrations. The effect of the malaxation temperature $\left(22,28\right.$ and $\left.34{ }^{\circ} \mathrm{C}\right)$ on the phenolic profile was studied for industrially extracted $c v$. Cobrançosa oils. Higher malaxation temperatures decreased the contents of the majority of the chromatographically detected compounds ( $P<0.05$, one-way ANOVA), enabling oils' differentiation. This decreasing trend was observed for hydroxytyrosol and tyrosol bound forms, determinant for the health claim, which were also negatively affected by temperature, despite revealing that all the industrially extracted oils tested supported the health claim. The observed constant free to bound forms ratio showed that the temperature range tested had a minor effect on bound-forms hydrolysis, being both free and bound forms equally affected by temperature.
\end{abstract}

\section{Introduction}

Consumers' awareness of the role of diet in health is sharply increasing (Olmo-García et al., 2019). Virgin olive oil (VOO) is the main external lipid ingredient of the Mediterranean diet, recognized worldwide as healthy and equilibrated. The positive health benefits associated with VOO consumption are mainly related to its richness in bioactive compounds like the olive phenolic compounds. Several phenolic compounds have been chromatographically identified in olive oils, including phenolic alcohols (e.g., hydroxytyrosol and tyrosol), secoiridoid derivatives (e.g., oleacein, oleocanthal, 4-(acetoxyethyl)1,2-dihydroxybenzene, oleuropein aglycone and its methylated form), phenolic acids and derivatives (e.g., vanillic acid and vanillin), lignans (e.g., pinoresinol and acetoxypinoresinol) and flavonoids (e.g., luteolin and apigenin) (Romani et al., 2019). NIR and mid-IR spectroscopy have also been used to assess the phenolic composition of olive oils (MoraRuiz et al., 2017). Over the past decade, compelling studies have been published reporting the benefic effects of VOO phenolic compounds on human cardiovascular health (Servili et al., 2014). This association is recognized by a health claim that refers that "olive oil polyphenols contribute to the protection of blood lipids from oxidative stress" for VOO that contained a minimum of $5 \mathrm{mg}$ of hydroxytyrosol and derivatives (e.g., oleuropein complex and tyrosol) per $20 \mathrm{~g}$ of olive oil (European Commission Regulation EU No 432/2012, 2012). Nevertheless, analytical concerns regarding the methodology that should support the claim have been raised (Mastralexi, Nenadis \& Tsimidou, 2014; Tsimidou, Nenadis, Servili, García-González \& Toschi, 2018). Since no specific analytical procedure was proposed for determining the target compounds that comprise a complex family of derivatives, and no adequate commercial standards are available, different results might be obtained from different analytical methodologies. The one adopted by the International Olive Council (IOC) for the determination of VOO biophenols (International Olive Council, 2017), gives a global quantification in terms of tyrosol equivalents and cannot support the new analytical need as it is (Tsimidou et al., 2019). Due to this fact, recently, Tsimidou et al. (2018) highlighted the need of consistent methods for the inclusion of all forms of hydroxytyrosol and tyrosol found in the oil to support the health claim requirement. Some authors (Mastralexi et al., 2014; Romero \& Brenes, 2012) have proposed to carry out a simple approach by measuring the amount of hydroxytyrosol and tyrosol released after hydrolysis of all bound forms as a way to simultaneously measure all the secoiridoid derivatives. However, taking into account the

\footnotetext{
* Corresponding authors.

E-mail addresses: sucasal@ff.up.pt (S. Casal), peres@ipb.pt (A.M. Peres).
} 
differences of the molecular weights between secoiridoids and their corresponding phenyl alcohols (i.e., between complex and simple phenols), correction factors were proposed for hydroxytyrosol (2.2) and tyrosol (2.5) (Mastralexi et al., 2014). As elucidated by Tsimidou et al. (2019), these correction factors were calculated as the ratio between the mean molecular mass ( $343 \mathrm{amu}$ ) of the most known bound forms of hydroxytyrosol and tyrosol (e.g., tyrosol glucoside, aldehydic form of ligstroside aglycone, dialdehyde form of ligstroside aglycone, aldehyde form of oleuropein aglycone, oleuropein aglycone, (carboxymethylated) dialdehyde form of oleuropein aglycone, (decarboxymethylated) dialdehyde form of oleuropein aglycone, (decarboxymethylated) aldehyde form of oleuropein aglycone) and the molecular mass of hydroxytyrosol (154 amu) and tyrosol (138 amu). Comparative studies showed satisfactory correlations between the amounts of hydroxytyrosol and tyrosol after hydrolysis and those determined by the IOC chromatographic method (Purcaro, Codony, Pizzale, Mariani, \& Conte, 2014) or the Folin-Ciocalteu colorimetric assay (ReboredoRodríguez, Valli, Bendini, Di Lecce, Simal-Gándara \& Toschi, 2016).

Olive oils' phenolic compounds play also key roles at the sensory level and on the oils' shelf-life. Different studies have been developed to optimize the extraction/production conditions (e.g., malaxation/extraction time-temperature conditions or controlling/removing oxygen supply during malaxation) aiming increasing the extraction yield of phenolics from the olive fruits (Angerosa, Mostallino, Basti, \& Vito, 2001; Gómez-Rico, Inarejos-García, Salvador, \& Fregapane, 2009; Guerrini et al., 2019; Lukić et al., 2017; Miho, Moral, López-González, Díez, \& Priego-Capote, 2020; Taticchi, Esposto, Veneziani, Urbani, Selvaggini \& Servili, 2013; Trapani et al., 2017). Besides the effects at the phenolic level, different malaxation temperatures/times also influenced the aroma quality of olive oils by changing the volatile fraction in terms of odorant series (Reboredo-Rodríguez, González-Barreiro, Cancho-Grande \& Simal-Gándara, 2014). Thus, this strategy would allow improving the overall chemical-sensory quality and ensuring that the legal requirements concerning the health claim are fulfilled (Guerrini et al., 2019; Lukić et al., 2017; Trapani et al., 2017). Nevertheless, the effect of olive oil extraction conditions on the phenolic profiles and contents is known to depend on the olive cultivar, the extraction plant scale (pilot versus industrial olive mills) and especially on the specific malaxation conditions (Clodoveo, 2012).

In summary, a limited number of studies evaluated the effect of the malaxation temperature (MT) on the phenolic composition of oils industrially extracted and no study assessed the possible effect of the MT on the fulfilment of the polyphenolic-related health claim. Regarding $c v$. Cobrançosa oils, one of the most widely cultivated cultivars in Portugal, no study has been performed at industrial scale neither to evaluate the MT effect on the extracted oils' phenolics or in the health claim fulfillment. In this context, it was intended to investigate the MT effect on the phenolic profile of $c v$. Cobrançosa olive oils industrially extracted and to assess the contribution to the fulfilment of the European Food Safety Authority health claim (European Food Safety Authority, 2011). Finally, the possible effect of the MT on the oil extraction yield was not considered, since it has been reported that MT between 20 and $40{ }^{\circ} \mathrm{C}$ do not significantly influence the extraction yield either at pilot or industrial scale (Guerrini et al., 2019; Ranalli, Contento, Schiavone, \& Simone, 2001; Veneziani et al., 2017).

\section{Materials and methods}

\subsection{Olive oil samples}

Olives from the cv. Cobrançosa were harvested in mid-November 2018 from an orchard located in Trás-os-Montes region (northeast Portugal). Fruits were harvested with a maturity stage (MI) between two and three, which corresponds to the fruit epidermis with red spots in less than half of the olive (MI 2) and the fruit epidermis red or purple in more than half of the olive (MI 3). Thus all fruits had a similar MI, allowing minimizing the influence of the MI on the olive oil composition. Oils were extracted in an industrial olive oil mill (OLIMONTES, Macedo de Cavaleiros, Portugal), at different MT (22, 28 and $\left.34{ }^{\circ} \mathrm{C}\right)$ during $60 \mathrm{~min}$ at 12 revolutions per minute (RPM), being used around $650 \mathrm{~kg}$ of olives for each extraction batch. These temperatures were chosen taking into account the MT range (from 20 up to $45^{\circ} \mathrm{C}$ ) usually reported in the literature and aiming to include oils obtained at temperatures below $27{ }^{\circ} \mathrm{C}$ (cold extraction) and at higher temperatures (Boselli, Di Lecce, Strabbioli, Pieralisi \& Frega, 2009; Guerrini et al., 2019; Ranalli et al., 2001; Veneziani et al., 2017). A modern two-phase unit mill was used (Alfa Laval, Italy). The line includes a hammer crusher, with a capacity of $5000 \mathrm{~kg}$ of olives per hour, and a sieve with $11 \mathrm{~mm}$ of diameter coupled with two malaxers (Type Gramula 700), each one with 4 bodies with a capacity of $650 \mathrm{~kg}$ of paste, a two-phase horizontal decanter centrifuge, with a capacity of $5000 \mathrm{~kg}$ of paste per hour, and a vertical centrifuge to wash and clean the olive oil (UVPX 507 AGT14). For each MT, an independent oil industrial extraction was performed being then collected six oil bottles throughout time (bottles were filled within a $10 \mathrm{~min}$ time-period before the oil entered the storage tank, ensuring some composition variability) and then closed at the end of the processing line. In the laboratory, olive oil samples were filtered through cellulose, to remove any suspended material. Olive oils were stored in amber glass bottles $(\sim 500 \mathrm{~mL})$ protected from light exposure. In total 18 olive oil bottles were obtained (collected in-line at the centrifuge output from 3 independent oil industrial extractions), corresponding to 6 oil subsamples for each of the 3 MT tested. Oils were analyzed within 5 months after extraction. All oils were classified as extra-virgin olive oils (free acidity lower than $0.8 \%$ of oleic acid, peroxide value lower than $20 \mathrm{mEq} \mathrm{O}_{2} / \mathrm{kg}$, extinction coefficients at 232 and $268 \mathrm{~nm}$ lower than 2.50 and 0.22 , respectively; fruity intensity greater than 0 and intensity of defects equal to 0 ) according to the European Union Commission Delegated Regulation (data not shown).

\subsubsection{Phenolic compounds analytical extraction and analysis by HPLC- $D A D$}

Phenolic compounds were analytically extracted according to the protocol of the IOC (International Olive Council, 2017), with minor modifications as previously described by Rodrigues et al. (2019). Briefly, to $0.4 \mathrm{~g}$ of olive oil, $25 \mu \mathrm{L}$ of a methanol/water $(80 / 20 \mathrm{v} / \mathrm{v})$ solution containing syringic acid $(0.15 \mathrm{mg} / \mathrm{mL}$; Sigma-Aldrich), used as an internal standard, were added, being then vortexed for $30 \mathrm{~s}$. Then $2.5 \mathrm{~mL}$ of the same methanol/water solution were added, and the solution vortexed for $30 \mathrm{~s}$, followed by the addition of $2.5 \mathrm{~mL}$ of hexane, aiming a better elimination of fat, being again agitated for $5 \mathrm{~min}$ in a horizontal mechanical shaker (Edmund Bühler $\mathrm{GmbH}$ ). Afterwards, the mixture was centrifuged at $5000 \mathrm{RPM}$ for $5 \mathrm{~min}$. The lower phase (hydrophilic) was transferred and filtered through a $0.22 \mu \mathrm{m}$ polyvinylidine fluoride (PVDF) microfilter. The solution was then taken to dryness under a gentle nitrogen stream $\left(40{ }^{\circ} \mathrm{C}\right)$ and immediately reconstituted with $100 \mu \mathrm{L}$ of methanol being ready for injection in the high-performance liquid chromatograph system (HPLC) coupled to a diode array detector (DAD). All oil samples (3 independent extractions (one for each MT) $\times 6$ oil bottles per MT) were analytically extracted in duplicate ( $\times 2$ analytical extractions per oil sample) and each extract was then injected twice (72 chromatographic assays).

The oils' phenolic profile was evaluated using a HPLC-DAD system from Jasco (Japan) with a data transmitter (LC-NetII/ADC), two integrated pumps (PU-4180), an auto-sampler (AS-4050), a column oven (ECOM Eco2000, Czech Republic), and the DAD (MD-4010). The separation was accomplished on a C18 reversed-phase column (Kinetex C18; particle size: $2.6 \mu \mathrm{m}$; pore size: $100 \AA$; LC length: $100 \mathrm{~mm}$; internal diameter: $3.00 \mathrm{~mm}$, Phenomenex), at $35{ }^{\circ} \mathrm{C}$, using an eluent gradient composed of water and acetonitrile, both with $0.1 \%$ of formic acid, at a flow rate of $0.8 \mathrm{~mL} / \mathrm{min}$. Peak identification was performed by comparing the retention times (RT) and UV/Vis spectra $(200-600 \mathrm{~nm})$ with those of pure standards (apigenin, apigenin 7-glucoside, 
Table 1

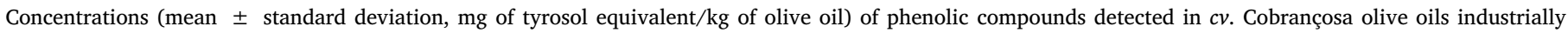
extracted at different malaxation temperatures $\left(22,28\right.$ and $34{ }^{\circ} \mathrm{C}$ ) and quantified according to the IOC method (HPLC-DAD).

\begin{tabular}{|c|c|c|c|c|c|}
\hline \multirow[t]{2}{*}{ Phenolic compounds } & \multicolumn{3}{|c|}{ Mean contents $(\mathrm{mg} / \mathrm{kg})$ at different malaxation temperatures } & \multirow[t]{2}{*}{$P$-value ${ }^{1}$} & \multirow[t]{2}{*}{$R$-Pearson ${ }^{2}$} \\
\hline & $22{ }^{\circ} \mathrm{C}$ & $28{ }^{\circ} \mathrm{C}$ & $34{ }^{\circ} \mathrm{C}$ & & \\
\hline \multicolumn{6}{|l|}{ Phenolic alcohols } \\
\hline Hydroxytyrosol & $1.9 \pm 0.3$ & $1.7 \pm 0.1$ & $1.8 \pm 0.4$ & 0.3725 & - \\
\hline Tyrosol & $2.5 \pm 0.3^{\mathrm{A}}$ & $2.8 \pm 0.2^{\mathrm{B}}$ & $2.6 \pm 0.2^{\mathrm{A}}$ & 0.0370 & - \\
\hline \multicolumn{6}{|c|}{ Dihydroxybenzoic derivative } \\
\hline Vanillic acid & $0.8 \pm 0.1^{\mathrm{A}}$ & $0.9 \pm 0.1^{\mathrm{B}}$ & $0.8 \pm 0.1^{\mathrm{A}}$ & $<0.0001$ & - \\
\hline \multicolumn{6}{|l|}{ Phenolic acids } \\
\hline p-Coumaric acid & $0.2 \pm 0.0^{\mathrm{A}}$ & $0.2 \pm 0.0^{\mathrm{A}}$ & $0.1 \pm 0.0^{\mathrm{B}}$ & $<0.0001$ & -0.703 \\
\hline Ferulic acid & $0.4 \pm 0.0^{\mathrm{A}}$ & $0.7 \pm 0.1^{\mathrm{B}}$ & $0.5 \pm 0.1^{\mathrm{A}}$ & $<0.0001$ & - \\
\hline Cinnamic acid & $10.7 \pm 1.4$ & $10.7 \pm 0.3$ & $10.8 \pm 1.0$ & 0.9677 & - \\
\hline \multicolumn{6}{|l|}{ Flavonoids } \\
\hline Luteolin & $9.9 \pm 0.4^{\mathrm{A}}$ & $6.7 \pm 0.3^{\mathrm{B}}$ & $7.0 \pm 0.4^{\mathrm{B}}$ & $<0.0001$ & -0.819 \\
\hline Apigenin & $7.8 \pm 0.2^{\mathrm{A}}$ & $7.0 \pm 0.2^{\mathrm{B}}$ & $7.3 \pm 0.3^{\mathrm{C}}$ & $<0.0001$ & -0.579 \\
\hline \multicolumn{6}{|l|}{ Secoiridoids aglycons } \\
\hline Oleuropein derivatives & $67.9 \pm 2.5^{\mathrm{A}}$ & $49.1 \pm 1.4^{\mathrm{B}}$ & $50.1 \pm 1.6^{\mathrm{B}}$ & $<0.0001$ & -0.842 \\
\hline Ligstroside derivatives & $62.4 \pm 1.5^{\mathrm{A}}$ & $52.8 \pm 1.0^{\mathrm{B}}$ & $54.6 \pm 1.7^{\mathrm{C}}$ & $<0.0001$ & -0.762 \\
\hline$\Sigma_{\text {dihydroxybenzoic derivatives }}$ & $0.8 \pm 0.1^{\mathrm{A}}$ & $0.9 \pm 0.1^{\mathrm{B}}$ & $0.8 \pm 0.1^{\mathrm{A}}$ & $<0.0001$ & - \\
\hline$\Sigma_{\text {flavonoids }}$ & $17.7 \pm 0.5^{\mathrm{A}}$ & $13.6 \pm 0.5^{\mathrm{B}}$ & $14.3 \pm 0.4^{\mathrm{C}}$ & $<0.0001$ & -0.779 \\
\hline$\Sigma_{\text {phenolic acids }}$ & $11.3 \pm 1.4$ & $11.6 \pm 0.28$ & $11.36 \pm 1.0$ & 0.7085 & - \\
\hline$\Sigma_{\text {phenolic alcohols }}$ & $4.44 \pm 0.6$ & $4.53 \pm 0.25$ & $4.33 \pm 0.5$ & 0.5735 & -0.536 \\
\hline$\Sigma_{\text {secoiridoids aglycons }}$ & $130.3 \pm 3.2^{\mathrm{A}}$ & $102.0 \pm 2.0^{\mathrm{B}}$ & $105.0 \pm 2.3^{\mathrm{B}}$ & $<0.0001$ & -0.818 \\
\hline$\Sigma_{\text {identified phenols }}{ }^{3}$ & $164.5 \pm 3.5^{\mathrm{A}}$ & $132.7 \pm 2.2^{\mathrm{B}}$ & $135.5 \pm 3.3^{\mathrm{B}}$ & $<0.0001$ & -0.823 \\
\hline$\Sigma_{\text {phenols }} 13<\mathrm{RT}<27 \min ^{4}$ & $282.0 \pm 11.1^{\mathrm{A}}$ & $270.6 \pm 6.6^{\mathrm{B}}$ & $285.6 \pm 5.0^{\mathrm{A}}$ & 0.0001 & - \\
\hline$\Sigma_{\text {other phenols }} 5$ & $123.3 \pm 10.6^{\mathrm{A}}$ & $144.3 \pm 5.3^{\mathrm{B}}$ & $155.8 \pm 3.6^{\mathrm{C}}$ & $<0.0001$ & +0.986 \\
\hline
\end{tabular}

${ }^{1} P$-values for the one-way ANOVA. Content means (for each MT, i.e., for each independent industrial oil extraction: $\mathrm{n}=24$; 6 olive oil samples $\times 2$ extrac-

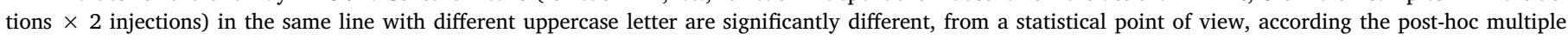
comparison Tukey test at $5 \%$ significance level.

2 R-Pearson coefficients: linear regression between concentration data and the malaxation temperature.

${ }^{3} \Sigma_{\text {identified phenols: }}$ sum of the contents (mg of tyrosol equivalent/kg oil) of each identified phenol (hydroxytyrosol, tyrosol, vanillic acid, p-coumaric acid, ferulic

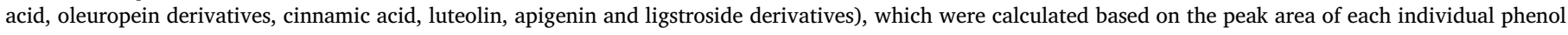
identified according to the IOC method.

${ }^{4} \Sigma_{\text {phenols } 13<\mathrm{RT}<27 \mathrm{~min}}$ : content (mg of tyrosol equivalent/kg oil) of all phenols (identified and unknown) calculated using the sum of the areas of all peaks with retention times (RT) between 13 and $27 \mathrm{~min}$.

$5 \Sigma_{\text {other phenols: }}$ content of the unknown (not identified) phenols that eluted with a RT between 13 and 27 min, calculated using the area data resulting from

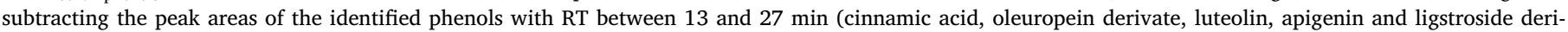
vatives) to the total area of all peaks within the same RT range.

hydroxytyrosol, luteolin and verbascoside, from ExtraSynthese; caffeic acid, cinnamic acid, ferulic acid, oleocanthal, oleuropein, p-coumaric acid, pinoresinol and vanillic acid, from Sigma-Aldrich; tyrosol (2-4hydroxyphenyl ethanol), from Fluka; oleacein, from Toronto Research Chemicals). Phenolic compounds identification followed the IOC method (International Olive Council, 2017) considering responses at different wavelengths as suggested by Torre-Carbot et al. (2005). Although commercial standards are available for some individual phenolic compounds, results were expressed as $\mathrm{mg}$ of tyrosol equivalents per $\mathrm{kg}$ of olive oil for each individual compound and the total phenols content, corresponding to the sum of the contents of all individual compounds quantified, using data obtained at $280 \mathrm{~nm}$ following the IOC guidelines (International Olive Council, 2017).

2.1.2. Total content of hydroxytyrosol and tyrosol derivatives: Acid hydrolysis of secoiridoids

Phenolic compounds from olive oils were also analytically extracted and analyzed according to the method proposed by Romero and Brenes (2012) with some modifications. From each oil sample, $0.1 \mathrm{~g}$ were weighed being added $0.006 \mathrm{mg}$ of internal standard $(40 \mu \mathrm{L}$ of a methanol/water (80:20, v/v) solution containing syringic acid (SigmaAldrich) at a concentration of $0.15 \mathrm{mg} / \mathrm{mL}$ ). Then, $2.5 \mathrm{~mL}$ of a hydrochloric acid methanol/water solution (80:20, v/v; $2 \mathrm{M}$ ) were added and agitated in a vortex during $30 \mathrm{~s}$. The solution was kept at $25^{\circ} \mathrm{C}$ for $6 \mathrm{~h}$ and, each $30 \mathrm{~min}$, the solution was agitated for $30 \mathrm{~s}$ in a vortex. After $6 \mathrm{~h}$ of hydrolysis, $2.5 \mathrm{~mL}$ of an acetonitrile/water solution (50:50 v/v) were added and transferred to Eppendorfs $(2 \mathrm{~mL})$ and centrifuged for $10 \mathrm{~min}$ at 13,000 RPM. After, $2 \mathrm{~mL}$ of the solution were removed, and $2 \mathrm{~mL}$ of $n$-hexane were added. The mixture was stirred for $30 \mathrm{~s}$ (vortex) and centrifuged for $3 \mathrm{~min}$ at $3500 \mathrm{RPM}$. Finally, the extract was filtered using a PVDF disposable filter $(0.22 \mu \mathrm{m})$ and chromatographically analyzed according to the same HPLC-DAD method and C18 column (previously described in section 2.2.1), at $280 \mathrm{~nm}$ during $20 \mathrm{~min}$. All samples were analytically extracted and injected in duplicate totalizing 72 chromatographic results (for the 3 independent industrial extractions, i.e., for the 3 MT: 3 MT $\times 6$ olive oil samples $\times 2$ analytical extractions $\times 2$ injections). The total hydroxytyrosol or tyrosol contents after hydrolysis were expressed as the individual sum in $\mathrm{mg}$ of hydroxytyrosol or tyrosol equivalents, respectively, per $\mathrm{kg}$ of oil. Hydroxytyrosol and tyrosol calibration curves $\left(R^{2}=0.9992\right.$ and 0.9990 , respectively) were prepared in methanol/water $(80: 20, \mathrm{v} / \mathrm{v})$ in a concentration range from 0.0005 to $0.02 \mathrm{mg} / \mathrm{mL}$. Because after hydrolysis only the tyrosol and hydroxytyrosol moieties are quantified, loosing information on the molecular weight of the original molecules, the original bound forms were estimated using the correction factors proposed in the literature for hydroxytyrosol (2.2) and tyrosol (2.5) (Mastralexi et al., 2014; Tsimidou et al., 2019).

\subsection{Statistical analysis}

Data were expressed as mean \pm standard deviation values. The statistical significance of the MT effect on the $c v$. Cobrançosa oils' 
phenolic profiles was assessed using one-way ANOVA followed, when appropriate, by the Tukey's post-hoc multi-comparison test. Also, when a significant effect was found, the Pearson correlation coefficient $(R$ Pearson) was determined to infer about the existence of a linear correlation between the oils' phenolic contents and the MT. All statistical analyzes were performed using the Sub-select (Cadima, Cerdeira, \& Minhoto, 2004) and MASS (Venables \& Ripley, 2002) packages of the open-source statistical program R (version 2.15.1), at a 5\% significance level.

\section{Results and discussion}

Malaxation consists of a continuous slow movement of the olive paste that provides an increase in "free oil" availability and helps oil droplets to merge into large drops. The efficiency of the malaxation depends on different conditions such as cultivar, fruit maturation, fruit quality, milling grade, amount of added water, time and temperature. Temperature is probably the factor that mostly influences the phenolic composition of the extracted oils (Di Giovacchino, Sestili \& Di Vincenzo, 2002). In this sense, the effect of the MT $\left(22,28\right.$ and $\left.34{ }^{\circ} \mathrm{C}\right)$ on the phenolic profile of $c v$. Cobrançosa olive oils and on the fulfilment of the phenolic-related health claim, was evaluated.

\subsection{Effect of the malaxation temperature on the oils' individual phenolic profiles assessed using the IOC method (HPLC-DAD)}

As shown in Table 1, the IOC chromatographic method (HPLC-DAD) allowed establishing the phenolic profiles of the $c v$. Cobrançosa olive oils extracted, which were found to be similar to those previously reported for other Portuguese olive oils (Rodrigues et al., 2019; Peres, Martins, Mourato, Vitorino, \& Ferreira-Dias, 2016). In this study, phenolic compounds of 5 different groups were detected and quantified. Table 1 shows the mean contents ( \pm standard deviations in $\mathrm{mg} / \mathrm{kg}$ oil, expressed in tyrosol equivalents) for phenolic alcohols (hydroxytyrosol and tyrosol), flavonoids (flavones: luteolin and apigenin), secoiridoids aglycons (oleuropein and ligstroside derivatives), phenolic acids (pcoumaric acid, ferulic acid and cinnamic acid) and dihydroxybenzoic derivatives (vanillic acid). The most abundant ones, for all MT, were the secoiridoids aglycons (oleuropein: 49-68 $\mathrm{mg} / \mathrm{kg}$; ligstroside: $53-62 \mathrm{mg} / \mathrm{kg})$, followed by cinnamic acid $(\sim 11 \mathrm{mg} / \mathrm{kg})$ and flavonoids (luteolin: $6.7-9.9 \mathrm{mg} / \mathrm{kg}$; apigenin: 7.0-7.8 mg/kg). Oppositely, hydroxytyrosol, tyrosol, vanillic acid, p-coumaric acid and ferulic acid were the less abundant phenolic compounds $(0.1-2.8 \mathrm{mg} / \mathrm{kg})$. The low amounts of the phenolic alcohols are in agreement with the studies of Peres et al. (2016) and Rodrigues et al. (2019) for Portuguese olive oils and could be tentatively attributed to the freshness of the olive oils (5 months after extraction) since hydroxytyrosol and tyrosol contents increase along the storage time due to the hydrolysis of secoiridoids (Brenes, García, García, \& Garrido, 2001), as well as to the different olive cultivars studied.

In general, the MT had a significant effect (Table 1) on the concentrations of most of the detected phenolics ( $P$-value $<0.05)$. However, it should be remarked that, from a practical point of view, for many phenolic compounds small content changes were observed and sometimes with slight different behaviors for the two most elevated temperatures. Mean contents usually decrease with the MT increase, pointing out the advantage of using lower MT on $c v$. Cobrançosa oils extraction. This effect was more pronounced for secoiridoids aglycons, being observed a reduction of approximately $26 \%$ and $12 \%$ of the total amounts of oleuropein ( $R$-Pearson $=-0.844)$ and ligstroside $(R$ Pearson $=-0.757$ ) derivatives, respectively, when the temperature raised from 22 to $28-34{ }^{\circ} \mathrm{C}$. This decrease could be attributed to the activation of endogenous oxidoreductase enzymes (Servili et al., 1998). Similar results were reported by Servili et al. (1998) and by Angerosa et al. (2001) for cvs. Coratina and Frantoio olive oils (MT from 25 to $35{ }^{\circ} \mathrm{C}$ ). Regarding flavonoids, an analogous decreasing trend was found when the MT increased from 22 to $28-34{ }^{\circ} \mathrm{C}$, being the concentration decrease more marked for luteolin (reduction of $\sim 29 \%, R$ Pearson $=-0.819$ ) compared to apigenin (reduction of $\sim 6 \%, R$ Pearson $=-0.579$ ). A similar decreasing trend with the temperature rise was reported by Lukić et al. (2017) for $c v$. Oblica oils extracted at 22 and $30{ }^{\circ} \mathrm{C}$ (luteolin: $4.10-3.63 \mathrm{mg} / \mathrm{kg}$; apigenin: $0.35-0.29 \mathrm{mg} / \mathrm{kg}$, respectively), even if the concentration ranges are cultivar dependent. This same trend was observed for the luteolin content of $c v$. Ayvalik oils when the MT raised from 27 to $37-47{ }^{\circ} \mathrm{C}$ (Jolayemi, Tokatli and Ozen, 2016). Contrary, other researchers reported that the flavonoids contents of olive oils were hardly affected by temperature (Gómez-Rico et al., 2009; Boselli et al., 2009; Torres et al., 2018). Concerning the phenolic alcohols, while the $12 \%$ of variation observed for the free hydroxytyrosol content was not statistically significant ( $P$ value $=0.3725$ ), the mean contents of tyrosol were significantly influenced by the MT ( $P$-value $=0.0370$ ), although no obvious trend could be found. Contrary, in some works, when the MT raised $\left(20-60{ }^{\circ} \mathrm{C}\right.$ ) the concentrations of some simple phenols (e.g., tyrosol and hydroxytyrosol) increased linearly, which was attributed to the hydrolysis of phenols with higher molecular weight (Parenti, Spugnoli, Masella, \& Calamai, 2008; Torres et al., 2018), being this trend more visible at higher temperatures, outside the range of the present study. Table 1 also shows that, in which concerns the phenolic acids, the mean contents of p-coumaric acid and ferulic acid were significantly affected by the MT increase $(P$-value $<0.05)$, although due to the low amounts found no practical inference could be established. On the other hand, the MT did not significantly influence the mean content of cinnamic acid $(\sim 10.7 \mathrm{mg} / \mathrm{kg} ; P$-value $=0.9677)$, with variations lower than $1 \%$. Oppositely, Torres et al. (2018) found that the increase of the MT had a negative impact on the content of phenolic acids of olive oils, although higher temperatures were tested $\left(20-60{ }^{\circ} \mathrm{C}\right)$. However, other studies reported a positive correlation between the MT and the concentration of phenols, which was justified by an enhanced release of olive phenols into the oil phase during the oil extraction at higher temperatures, providing that they were kept below $35^{\circ} \mathrm{C}$ (Boselli et al., 2009; Ranalli et al., 2001). Lastly, it should be remarked that the MT (Table 1) also had a significant effect $(P$-value $<0.05)$ on the total mean content of the identified phenols and of the not identified phenols, although an opposite effect was observed. The total amount of the identified phenols decreased $19 \%$ (from 164.5 to $132.7-135.5 \mathrm{mg} / \mathrm{kg}$ for 22 and $28-34{ }^{\circ} \mathrm{C}$, respectively; $R$-Pearson $=-0.823$ ) with the increase of the MT and the total amount of the non-identified phenols increased $26 \%$ (from 123.3 to $155.8 \mathrm{mg} / \mathrm{kg}$ for 22 and $34{ }^{\circ} \mathrm{C}$, respectively; $R$-Pearson $=+0.986$ ). The literature data pointed out that the total phenols contents of oils strongly increase with the raise of the MT $\left(20-45{ }^{\circ} \mathrm{C}\right.$ ) (Boselli et al., 2009; Guerrini et al., 2019; Lukić et al., 2017; Veneziani et al., 2017). As hypothesized by Taticchi et al. (2013), the increase of phenols concentration could be partly due to the lower temperature stability of the polyphenoloxidase (PPO) enzyme and its partial deactivation at higher temperatures, although this finding was shown to be a strongly cultivar-dependent feature. In addition to the decreasing of the PPO activity, higher MT could also enhance the release of phenols from the cell wall polysaccharides and from other olive tissues (Taticchi et al., 2013), promoting the increase of the partition coefficient of phenols between the oil and water phases in the olive paste (Gómez-Rico et al., 2009) leading to an increase of the phenol concentrations in the olive oils. Nevertheless, some studies showed that the total content of phenols increased with the MT up to $30{ }^{\circ} \mathrm{C}$; decreasing at higher temperatures (Parenti et al., 2008) or ceasing to increase (Ranalli et al., 2001). It should be noticed that the total phenol contents of the $c v$. Cobrançosa oils were much lower than those reported by Veneziani et al. (2017) for cvs. Coratina, Ottobratica and Peranzana oils (970.8-1327.8; 436.2-547.5 and 376.4-456.5 mg/kg, respectively), extracted at 25 and $30{ }^{\circ} \mathrm{C}$; or, by Lukić et al. (2017) for $c v$. Oblica oils extracted at 22 and $30{ }^{\circ} \mathrm{C}$ (594 and $804 \mathrm{mg} / \mathrm{kg}$, respectively), which could be partially attributed to the olive cultivar effect plus the applied 
specific malaxation conditions. Besides the above-mentioned discussion, one important issue that should be taken into account is the extraction equipment used, being reported different oils' compositions depending if the extraction was made on a laboratory, pilot or industrial olive mill. Indeed, data from extractions performed at laboratory scale are not always useful to predict the composition of oils obtained with an industrial olive mill (Di Giovacchino et al., 2002). Since the phenolic profiles of olive oils and their individual contents depend on the extraction conditions (e.g., temperature-time of malaxation), as well as on the olive cultivar, fruit ripening stage, agro-climatic conditions, crop year and geographical origin, in the present study, all oils were extracted from olives of the same cultivar, grown under the same agronomic practices and climatic conditions, being collected with the same maturation index, extracted in the same industrial plant, ensuring that only the MT varied.

\subsection{Effect of the malaxation temperature on the total content of hydroxytyrosol and tyrosol derivatives and health claim evaluation}

The IOC chromatographic method is time-consuming, being difficult to ascertain the true amount of compounds individually due to the complexity of compounds derived from tyrosol and hydroxytyrosol and the inexistence of commercial standards. So, simpler analytical techniques have been proposed to evaluate the secoiridoidic fraction of the oils (Mastralexi et al., 2014). This latter strategy is based on the hydrolysis of the phenolic bound forms and, although not providing a deep information regarding the specific oils' phenolic profile, allows a fast estimation of the hydroxytyrosol and tyrosol derivatives contents. It has been widely applied (Bartella, Mazzotti, Napoli, Sindona, \& Di Donna, 2018; Bellumori et al., 2019; Mastralexi et al., 2014; OlmoGarcía et al., 2019; Purcaro et al., 2014; Reboredo-Rodríguez et al., 2016; Romero \& Brenes, 2012) and allowed assessing the oils' health claim fulfillment (European Commission Regulation EU No 432/2012, 2012), providing that conversion factors are applied to estimate the amounts of the original molecules before hydrolysis.

Therefore, the contents of hydroxytyrosol and tyrosol derivatives of $c v$. Cobrançosa oils, extracted at the 22,28 and $34^{\circ} \mathrm{C}$, were quantified by HPLC-DAD after the acid hydrolysis of the polar fraction. Acid hydrolysis promoted the release of hydroxytyrosol and tyrosol from their conjugated forms, providing a realistic evaluation of the potential secoiridoid pool of the oils (Bellumori et al., 2019) and establishing the nutritional quality of the oils (Bartella et al., 2018). Table 2 presents the contents of hydroxytyrosol and tyrosol (mg/20 g oil) determined without and with the use of the mass differences correction factors to estimate the amounts of the related derivatives (Mastralexi et al., 2014).

In which concerns the uncorrected concentrations (no use of the mass differences correction factors), $c v$. Cobrançosa oils extracted at lower MT $\left(22\right.$ or $28{ }^{\circ} \mathrm{C}$ ) contained greater amounts of hydroxytyrosol and tyrosol derivatives, as well as of the respective sum ( $P$-value $<$ 0.05), than oils extracted at $34{ }^{\circ} \mathrm{C}$ (more 4-5\%). A linear decrease with the temperature increase $(-0.967 \leq R$-Pearson $\leq-0.930)$ was observed, being more sharped for tyrosol derivatives, strengthening the hypothesis that oils' phenolic composition is enhanced by lower MT. Then again, the tyrosol derivatives contents $(2.32-2.43 \mathrm{mg} / 20 \mathrm{~g}$ oil) were higher than the hydroxytyrosol derivatives $(1.51-1.57 \mathrm{mg} / 20 \mathrm{~g}$ oil), which is in agreement with the literature data for commercial Argentinian (Castelli, Bianco, \& Mizutamari, 2018) and Portuguese oils (Pereira, Freitas, Cabrita, \& Garcia, 2020). The ratio of free to bound tyrosol and hydroxytyrosol derivatives was further evaluated since it can be an effective measure of the impact of the MT on the phenolic forms that contribute to the health claim. For the $c v$. Cobrançosa oils industrially extracted the ratio of free (hydroxytyrosol contents corrected according to Bellumori et al. (2019) plus tyrosol contents), to bound (amounts after acid hydrolysis) forms was approximately constant (free/bound ratio ranging from $0.0188 \pm 0.002$ and $0.0197 \pm 0.001$ for $22-34{ }^{\circ} \mathrm{C}$ ). This finding revealed, that for the studied $c v$. Cobrançosa oils under the extraction conditions evaluated, both free and bound forms were similarly affected by the MT (ranging from 22 to $34^{\circ} \mathrm{C}$ ), pointing out that the temperature range tested had a minor effect on bound-forms hydrolysis.

Based on the total amounts of hydroxytyrosol and tyrosol derivatives, calculated without the correction factors $(3.83-3.99 \mathrm{mg} / 20 \mathrm{~g}$ ), none of the oils would fulfil the health claim (European Commission Regulation EU No 432/2012, 2012). These values are lower than those reported by Pereira et al. (2020) for $c v$. Cobrançosa oils extracted in a pilot plant at $25^{\circ} \mathrm{C}(7.94 \pm 3.77 \mathrm{mg} / 20 \mathrm{mg})$. However, as proposed in the literature (Castelli et al., 2018; Mastralexi et al., 2014; Tsimidou et al., 2019), if the correction factors were used, all $c v$. Cobrançosa oils met the health claim, with total amounts of both phenolic alcohols derivatives greater than $5 \mathrm{mg} / 20 \mathrm{~g}$ oil $(9.52,9.43$ and $9.13 \mathrm{mg} / 20 \mathrm{~g}$ oil for 22,28 and $34{ }^{\circ} \mathrm{C}$, respectively). In general, our results are in agreement with the literature that reported a wide range of contents (tyrosol derivatives: $0.2-11 \mathrm{mg} / 20 \mathrm{~g}$ oil, hydroxytyrosol derivatives:

Table 2

Amounts of hydroxytyrosol, tyrosol and sum of both compounds (mean \pm standard deviation, $\mathrm{mg} / 20 \mathrm{~g}$ of oil) found in $c v$. Cobrançosa olive oils industrially extracted at different malaxation temperatures $\left(22,28\right.$ and $\left.34^{\circ} \mathrm{C}\right)$, after the secoiridoids' acid hydrolysis and determined by HPLC-DAD ( $\left.280 \mathrm{~nm}\right)$.

\begin{tabular}{|c|c|c|c|c|c|c|}
\hline \multirow[b]{3}{*}{ Mass correction } & \multirow[b]{3}{*}{ Phenolic compounds after acid hydrolysis } & \multicolumn{4}{|c|}{ Malaxation Temperature } & \multirow{3}{*}{$R$-Pearson ${ }^{2}$} \\
\hline & & \multicolumn{3}{|c|}{ Content $(\mathrm{mg} / 20 \mathrm{~g}$ of oil) } & \multirow[t]{2}{*}{$P$-value ${ }^{1}$} & \\
\hline & & $22{ }^{\circ} \mathrm{C}$ & $28{ }^{\circ} \mathrm{C}$ & $34{ }^{\circ} \mathrm{C}$ & & \\
\hline $\mathrm{No}^{3}$ & Hydroxytyrosol & $1.57 \pm 0.03^{\mathrm{A}}$ & $1.56 \pm 0.04^{\mathrm{A}}$ & $1.51 \pm 0.04^{\mathrm{B}}$ & 0.0004 & -0.930 \\
\hline Yes $^{4}$ & Hydroxytyrosol & $3.45 \pm 0.07^{\mathrm{A}}$ & $3.43 \pm 0.08^{\mathrm{A}}$ & $3.32 \pm 0.08^{\mathrm{B}}$ & & \\
\hline $\mathrm{No}^{3}$ & Tyrosol & $2.43 \pm 0.05^{\mathrm{A}}$ & $2.40 \pm 0.04^{\mathrm{A}}$ & $2.32 \pm 0.04^{\mathrm{B}}$ & $<0.0001$ & -0.967 \\
\hline Yes $^{4}$ & Tyrosol & $6.07 \pm 0.13^{\mathrm{A}}$ & $6.00 \pm 0.10^{\mathrm{A}}$ & $5.81 \pm 0.09^{\mathrm{B}}$ & & \\
\hline $\mathrm{No}^{3}$ & Hydroxytyrosol + Tyrosol & $3.99 \pm 0.08^{\mathrm{A}}$ & $3.96 \pm 0.07^{\mathrm{A}}$ & $3.83 \pm 0.07^{\mathrm{B}}$ & $<0.0001$ & -0.955 \\
\hline Yes $^{4}$ & Hydroxytyrosol + Tyrosol & $9.52 \pm 0.20^{\mathrm{A}}$ & $9.43 \pm 0.17^{\mathrm{A}}$ & $9.13 \pm 0.16^{\mathrm{B}}$ & & \\
\hline
\end{tabular}

1 P-values for the one-way ANOVA. Content means $(n=24$; for each independent industrial oil extraction corresponding to each MT: 6 olive oil samples $\times 2$

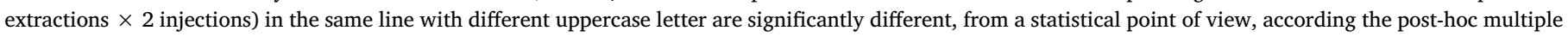
comparison Tukey test at $5 \%$ significance level.

$2 R$-Pearson coefficients: linear regression between concentration data and the malaxation temperature.

${ }^{3}$ Health claim regarding the protection of blood lipids from oxidative stress (European Commission Regulation EU No 432/2012, 2012): contents given as mg/

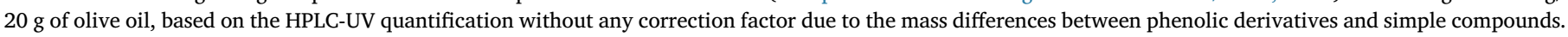

${ }^{4}$ Health claim regarding the protection of blood lipids from oxidative stress (European Commission Regulation EU No 432/2012, 2012): contents given as mg/

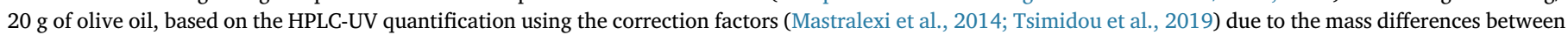
phenolic derivatives and simple compounds (hydroxytyrosol: 2.2; and, tyrosol: 2.5). 
0-9 mg/20 g oil, derivatives sum: $0-20 \mathrm{mg} / 20 \mathrm{~g}$ oil), which could be related with the olive cultivar, extraction conditions, geographical origin and storage time and conditions (Bartella et al., 2018; Bellumori et al., 2019; Castelli et al., 2018; Mastralexi et al., 2014; Olmo-García et al., 2019; Pereira et al., 2020; Purcaro et al., 2014; ReboredoRodríguez et al., 2016; Tsimidou et al., 2019). Also, if the secoiridoids contents determined according to the IOC method and reported on a tyrosol basis (Table 1) were re-calculated using the correction factors (Mastralexi et al., 2014), a similar finding would be achieved, confirming that all $\mathrm{cv}$. Cobrançosa oils industrially extracted fulfilled the health claim $(6.32 \pm 0.16,5.02 \pm 0.10$ and $5.14 \pm 0.12 \mathrm{mg} / 20 \mathrm{~g}$ of oil for 22,28 and $34{ }^{\circ} \mathrm{C}$, respectively). Nevertheless, the health claim related values were lower (minus 34-47\%) than those determined based on the acid hydrolysis method, indicative that most likely no universal conversion factor can give a true estimation on the health potential.

Finally, the results, in line with the literature, clearly show that the quantification of hydroxytyrosol and tyrosol derivatives by HPLC-DAD after acid hydrolysis of the oils' polar analytical extracts could be a suitable simple analytical strategy to be implemented by olive oil producers to evaluate their amounts in oils extracted at industrial scale allowing assessing the fulfillment of the desired health claim. In fact, when a detailed and comprehensive phenolic profiling is not required, the simpler acid hydrolysis method can be a suitable alternative. Additionally, it shows that under all the conditions assayed the health claim could be labelled contributing to enhance the olive oils' commercial competitiveness.

\section{Conclusions}

The phenolic profiles of $c v$. Cobrançosa olive oils, industrially extracted at 22, 28 and $34{ }^{\circ} \mathrm{C}$, established according to the IOC method showed that all oils contained dihydroxybenzoic derivatives, flavonoids, phenolic acids, phenolic alcohols, and secoiridoids aglycons, being the latter the most abundant. In general, for the main phenolic compounds, a significant decrease in their abundance was found with the increase of the MT. A similar decreasing significant trend was observed for the total phenol contents. Also, it was found that, despite the minor differences observed, the phenolic profile of $c v$. Cobrançosa oils extracted at $22{ }^{\circ} \mathrm{C}$ was distinct from that obtained at 28 and $34{ }^{\circ} \mathrm{C}$. In contrast, the acid hydrolysis-chromatographic approach proved to be a fast and satisfactory analytical method for quantifying the amounts of hydroxytyrosol and tyrosol derivatives allowing a straightforward procedure for evaluating the oils' health claim fulfillment. However, if a detailed understanding of the phenolic profile is needed, namely regarding flavonoids, lignans and phenolic acids, the methodology recommended by the IOC or other similar method must be adopted. The results of the acid hydrolysis method together with the use of the mass difference correction factors allowed concluding that all $c v$. Cobrançosa oils met the health claim ensuring that their daily intake would contribute to the protection of blood lipids from oxidative stress. Furthermore, it was found that oils extracted at 22 or $28{ }^{\circ} \mathrm{C}$ presented the higher contents of total hydroxytyrosol and tyrosol derivatives. So, in conclusion, the present study suggested that oils extracted at lower temperatures $\left(22^{\circ} \mathrm{C}\right)$ are, in general, richer in phenolic compounds and so may promote the health benefits related to their consumption, requiring the lowest daily intake (approximately $10 \mathrm{~g}$ per day) to fulfill the health claim.

\section{Declaration of Competing Interest}

The authors declare that they have no known competing financial interests or personal relationships that could have appeared to influence the work reported in this paper.

\section{Acknowledgments}

The authors are grateful to the Foundation for Science and Technology (FCT, Portugal) for financial support by national funds FCT/MCTES to CIMO (UIDB/00690/2020), to CEB (UIDB/04469/ 2020), to REQUIMTE-LAQV (UIDB/50006/2020) and to BioTecNorte operation (NORTE-01-0145-FEDER-000004) funded by the European Regional Development Fund under the scope of Norte2020 - Programa Operacional Regional do Norte. Ítala Marx acknowledges the Ph.D. research grant (SFRH/BD/137283/2018) provided by FCT. Nuno Rodrigues thanks the National funding by FCT- Foundation for Science and Technology, P.I., through the institutional scientific employment program-contract.

\section{Appendix A. Supplementary data}

Supplementary data to this article can be found online at https:// doi.org/10.1016/j.foodchem.2020.127726.

\section{References}

Angerosa, F., Mostallino, R., Basti, C., \& Vito, R. (2001). Influence of malaxation temperature and time on the quality of virgin olive oils. Food Chemistry, 72, 19-28. https://doi.org/10.1016/S0308-8146(00)00194-1.

Bartella, L., Mazzotti, F., Napoli, A., Sindona, G., \& Di Donna, L. (2018). A comprehensive evaluation of tyrosol and hydroxytyrosol derivatives in extra virgin olive oil by microwave-assisted hydrolysis and HPLC-MS/MS. Analytical and Bioanalytical Chemistry, 410, 2193-2201. https://doi.org/10.1007/s00216-018-0885-1.

Bellumori, M., Cecchi, L., Innocenti, M., Clodoveo, M. L., Corbo, F., \& Mulinacci, N. (2019). The EFSA health claim on olive oil polyphenols: Acid hydrolysis validation and total hydroxytyrosol and tyrosol determination in Italian virgin olive oils. Molecules, 24, 1-16. https://doi.org/10.3390/molecules24112179.

Boselli, E., Di Lecce, G., Strabbioli, R., Pieralisi, G., \& Frega, N. G. (2009). Are virgin olive oils obtained below $27^{\circ} \mathrm{C}$ better than those produced at higher temperatures? LWT Food Science and Technology, 42(3), 748-757. https://doi.org/10.1016/j.lwt.2008.09. 018.

Brenes, M., García, A., García, P., \& Garrido, A. (2001). Acid hydrolysis of secoiridoid aglycons during storage of virgin olive oil. Journal of Agricultural and Food Chemistry, 49, 5609-5614. https://doi.org/10.1021/jf0107860.

Cadima, J., Cerdeira, J. O., \& Minhoto, M. (2004). Computational aspects of algorithms for variable selection in the context of principal components. Computational Statistics \& Data Analysis, 47(2), 225-236. https://doi.org/10.1016/j.csda.2003.11.001.

Castelli, G., Bianco, I. D., \& Mizutamari, R. K. (2018). Polyphenol content in Argentinean commercial extra virgin olive oil. European Journal of Lipid Science and Technology, 120, 1-5. https://doi.org/10.1002/ejlt.201800124.

Clodoveo, M. L. (2012). Malaxation: influence on virgin olive oil quality. Past, present and future - An overview. Trends in Food Science and Technology, 25(1), 13-23. https://doi.org/10.1016/j.tifs.2011.11.004.

Di Giovacchino, L., Sestili, S., \& Di Vincenzo, D. (2002). Influence of olive processing on virgin olive oil quality. European Journal of Lipid Science and Technology, 104, 587-601. https://doi.org/10.1002/1438-9312(200210)104:9/10<587::AIDEJLT587 > 3.0.CO;2-M.

European Commission Regulation (EU) No 432/2012 (2012). Establishing a List of Permitted Health Claims Made on Foods Other Than Those Referring to the Reduction of Disease Risk and to Children's Development and Health. Official Journal of the European Union, 1-40, L136.

European Food Safety Authority (2011). EFSA panel on dietetic products, nutrition and allergies. scientific opinion on the substantiation of health claims related to polyphenols in olive oil and protection of LDL particles from oxidative damage. EFSA Journal, 9, 1-25. http://www.efsa.europa.eu/en/efsajournal/pub/2033.htm.

Gómez-Rico, A., Inarejos-García, A. M., Salvador, M. D., \& Fregapane, G. (2009). Effect of malaxation conditions on phenol and volatile profiles in olive paste and the corresponding virgin olive oils (Olea europaea L. Cv. cornicabra). Journal of Agricultural and Food Chemistry, 57, 3587-3595. https://doi.org/10.1021/jf803505w.

Guerrini, L., Masella, P., Angeloni, G., Zanoni, B., Breschi, C., Calamai, L., \& Parenti, A. (2019). The effect of an increase in paste temperature between malaxation and centrifugation on olive oil quality and yield: Preliminary Results. Italian Journal of Food Science, 31, 451-458. https://doi.org/10.14674/IJFS-1393.

International Olive Council (2017). Determination of Biophenols in Olive Oils by HPLC, COI/T.20/Doc No 29/Rev.1. 29, 1-8.

Jolayemi, O. S., Tokatli, F., \& Ozen, B. (2016). Effects of malaxation temperature and harvest time on the chemical characteristics of olive oils. Food Chemistry, 211, 776-783. https://doi.org/10.1016/j.foodchem.2016.05.134.

Lukić, I., Žanetić, M., Špika, M. J., Lukić, M., Koprivnjak, O., \& Bubola, K. B. (2017). Complex interactive effects of ripening degree, malaxation duration and temperature on Oblica cv. virgin olive oil phenols, volatiles and sensory quality. Food Chemistry, 232, 610-620. https://doi.org/10.1016/j.foodchem.2017.04.047.

Mastralexi, A., Nenadis, N., \& Tsimidou, M. Z. (2014). Addressing analytical requirements to support health claims on "olive oil polyphenols" (EC regulation 432/2012). Journal 
of Agricultural and Food Chemistry, 62, 2459-2461. https://doi.org/10.1021/ jf5005918.

Miho, H., Moral, J., López-González, M. A., Díez, C. M., \& Priego-Capote, F. (2020). The phenolic profile of virgin olive oil is influenced by malaxation conditions and determines the oxidative stability. Food Chemistry, 314, Article 126183. https://doi.org/ 10.1016/j.foodchem.2020.126183.

Mora-Ruiz, M. E., Reboredo-Rodríguez, P., Salvador, M. D., González-Barreiro, C., Cancho-Grande, B., Simal-Gándara, J., \& Fregapane, G. (2017). Assessment of polar phenolic compounds of virgin olive oil by NIR and mid-IR spectroscopy and their impact on quality. European Journal of Lipid Science and Technology, 119(1), 1600099. https://doi.org/10.1002/ejlt.201600099.

Olmo-García, L., Fernández-Fernández, C., Hidalgo, A., Vílchez, P., Fernández-Gutiérrez, A., Marchal, R., \& Carrasco-Pancorbo, A. (2019). Evaluating the reliability of specific and global methods to assess the phenolic content of virgin olive oil: Do they drive to equivalent results?. Journal of Chromatography A, 1585, 56-69. https://doi.org/10. 1016/j.chroma.2018.11.031.

Parenti, A., Spugnoli, P., Masella, P., \& Calamai, L. (2008). The effect of malaxation temperature on the virgin olive oil phenolic profile under laboratory-scale conditions. European Journal of Lipid Science and Technology, 110, 735-741. https://doi.org/10. 1002/ejlt.200700307.

Pereira, C., Freitas, A. M. C., Cabrita, M. J., \& Garcia, R. (2020). Assessing tyrosol and hydroxytyrosol in Portuguese monovarietal olive oils: Revealing the nutraceutical potential by a combined spectroscopic and chromatographic techniques - Based approach. LWT- Food Science and Technology, 118, Article 108797. https://doi.org/ 10.1016/j.lwt.2019.108797.

Peres, F., Martins, L. L., Mourato, M., Vitorino, C., \& Ferreira-Dias, S. (2016). Bioactive compounds of Portuguese virgin olive oils discriminate cultivar and ripening stage. JAOCS Journal of the American Oil Chemists' Society, 93, 1137-1147. https://doi.org/ 10.1007/s11746-016-2848-z.

Purcaro, G., Codony, R., Pizzale, L., Mariani, C., \& Conte, L. (2014). Evaluation of total hydroxytyrosol and tyrosol in extra virgin olive oils. European Journal of Lipid Science and Technology, 116, 805-811. https://doi.org/10.1002/ejlt.201300420.

Ranalli, A., Contento, S., Schiavone, C., \& Simone, N. (2001). Malaxing temperature affects volatile and phenol composition as well as other analytical features of virgin olive oil. European Journal of Lipid Science and Technology, 103, 228-238. https://doi. org/10.1002/1438-9312(200104)103:4<228::AID-EJLT228 > 3.0.CO.2-7.

Reboredo-Rodríguez, P., González-Barreiro, C., Cancho-Grande, B., \& Simal-Gándara, J. (2014). Improvements in the malaxation process to enhance the aroma quality of extra virgin olive oils. Food Chemistry, 158, 534-545. https://doi.org/10.1016/j. foodchem.2014.02.140.

Reboredo-Rodríguez, P., Valli, E., Bendini, A., Di Lecce, G., Simal-Gándara, J., \& Toschi, T. G. (2016). A widely used spectrophotometric assay to quantify olive oil biophenols according to the health claim (EU Reg. 432/2012). European Journal of Lipid Science and Technology, 118, 1593-1599. https://doi.org/10.1002/ejlt.201500313.

Rodrigues, N., Casal, S., Pinho, T., Peres, A. M., Bento, A., Baptista, P., \& Pereira, J. A. (2019). Ancient olive trees as a source of olive oils rich in phenolic compounds. Food Chemistry, 276, 231-239. https://doi.org/10.1016/j.foodchem.2018.09.106.

Romani, A., Ieri, F., Urciuoli, S., Noce, A., Marrone, G., Nediani, C., \& Bernini, R. (2019).
Health Effects of Phenolic Compounds Found in Extra-Virgin Olive Oil, By-Products, and Leaf of Olea europaea L. Nutrients, 11, 1-33. https://doi.org/10.3390/ nu11081776.

Romero, C., \& Brenes, M. (2012). Analysis of total contents of hydroxytyrosol and tyrosol in olive oils. Journal of Agricultural and Food Chemistry, 60, 9017-9022. https://doi. org/10.1021/jf3026666.

Servili, M., Baldioli, M., Selvaggini, R., Mariotti, F., Federici, E., \& Montedoro, G. F. (1998). Effect of Malaxation Under N2 Flush on Phenolic and Volatile Compounds of Virgin Olive Oil. in Advances in plant Lipid Research, Proceedings of the 13th International Symposium on Plant Lipids, 307-310.

Servili, M., Sordini, B., Esposto, S., Urbani, S., Veneziani, G., Di Maio, I., ... Taticchi, A. (2014). Biological activities of phenolic compounds of extra virgin olive oil. Antioxidants, 3, 1-23. https://doi.org/10.3390/antiox3010001.

Taticchi, A., Esposto, S., Veneziani, G., Urbani, S., Selvaggini, R., \& Servili, M. (2013). The influence of the malaxation temperature on the activity of polyphenoloxidase and peroxidase and on the phenolic composition of virgin olive oil. Food Chemistry, 136, 975-983. https://doi.org/10.1016/j.foodchem.2012.08.071.

Torre-Carbot, K., Jauregui, O., Gimeno, E., Castellote, A. I., Lamuela-Raventós, R. M., \& Carmen López-Sabater, M. (2005). Characterization and quantification of phenolic compounds in olive oils by solid-phase extraction, HPLC-DAD, and HPLC-MS/MS. Journal of Agricultural and Food Chemistry, 53, 4331-4340. https://doi.org/10.1021/ jf0501948.

Torres, A., Espínola, F., Moya, M., Alcalá, S., Vidal, A. M., \& Castro, E. (2018). Assessment of phenolic compounds in virgin olive oil by response surface methodology with particular focus on flavonoids and lignans. LWT - Food Science and Technology, 90, 22-30. https://doi.org/10.1016/j.lwt.2017.12.003.

Trapani, S., Breschi, C., Cecchi, L., Guerrini, L., Mulinacci, N., Parenti, A., ... Zanoni, B. (2017). Indirect indices of oxidative damage to phenolic compounds for the implementation of olive paste malaxation optimization charts. Journal of Food Engineering, 207, 24-34. https://doi.org/10.1016/j.jfoodeng.2017.03.012.

Tsimidou, M. Z., Nenadis, N., Mastralexi, A., Servili, M., Butinar, B., Vichi, S., ... Toschi, T. G. (2019). Toward a harmonized and standardized protocol for the determination of total hydroxytyrosol and tyrosol content in virgin olive oil (VOO). The pros of a fit for the purpose ultra high-performance liquid chromatography (UHPLC) Procedure. Molecules, 24, 1-16. https://doi.org/10.3390/molecules24132429.

Tsimidou, M. Z., Nenadis, N., Servili, M., García-González, D. L., \& Toschi, G. T. (2018). Why tyrosol derivatives have to be quantified in the calculation of "olive oil polyphenols" content to support the health claim provisioned in the EC Reg. 432/2012. European Journal of Lipid Science and Technology, 120, 1-6. https://doi.org/10.1002/ ejlt.201800098.

Venables, W. N., \& Ripley, R. B. (2002). Modern applied statistics with S (statistics and computing) (4th ed.). New York: Springerhttps://doi.org/10.1007/978-0-38721706-2.

Veneziani, G., Esposto, S., Taticchi, A., Urbani, S., Selvaggini, R., Di Maio, I., ... Servili, M. (2017). Cooling treatment of olive paste during the oil processing: Impact on the yield and extra virgin olive oil quality. Food Chemistry, 221, 107-113. https://doi. org/10.1016/j.foodchem.2016.10.067. 